\title{
Potential Antiviral Activity of Statins Against SARS-CoV-2 Through Inhibition of the Main Viral Protease
}

\author{
Milad Shirvaliloo ${ }^{1}$ \\ ${ }^{1}$ Tabriz University of Medical Sciences
}

June 23, 2020

Dear Editor,

Almost half a year into the COVID-19 pandemic, the one lesson that we all have probably learned is that the novel coronavirus infection is not an issue to be taken lightly. With millions of confirmed cases, and thousands of death reports, it has become absolutely clear that there is no magic bullet at our disposal in case of COVID-19. Due to the lack of medications specific to the etiological cause of COVID19, i.e., SARS-CoV-2, clinicians in all parts of the world are left with an exceedingly limited array of therapeutic options, that is mostly restricted to supportive and palliative care (Sheervalilou et al., 2020).

To this date, several treatment modalities and numerous drugs of different classes have been evaluated for their efficacy in treatment of the novel viral pneumonia, with only a few of them showing promising clinical results. Despite the clinical administration of certain classes of drugs such as the antimalarial hydroxychloroquine, ACE inhibitors, and lopinavir/ritonavir, there is still inadequate evidence that would support their efficacy in treatment of COVID-19 (Sheervalilou et al., 2020). However, according to the most recent findings, statins or cholesterol lowering drugs might be of unappreciated therapeutic value in the ongoing fight against COVID-19. This is a possibility of great importance for individuals with atherosclerotic cardiovascular disease (ASCVD), who are most commonly treated with statins, and of course, fall into the category with an increased susceptibility to severe COVID-19 (Reiner et al., 2020).

Traditionally known as cholesterol lowering drugs, statins do more than simply regulating the level of cholesterol in the blood, as they possess anti-inflammatory properties, and can potentially counteract the pathologies that might result in thrombotic events, e.g., septic shock (Angus \& van der Poll, 2013). With their immune-modulatory functions, statins are thought to have the potency to interfere with the infectivity of several strains of viruses, as they can very well inhibit the processing of viral glycoproteins by disrupting the activity of the main viral protease. Impairment of the protease activity, and the consequential fall in the amount of processed glycoproteins are postulated to be the mechanisms behind the unappreciated antiviral effects of statins (Shrivastava-Ranjan et al., 2018).

In the case of coronaviruses, the main protease or "Mpro" is a cleaving enzyme, that leads a prominent role in the maturation of viral glycoproteins. The enzyme has been indicated to be a potential target of antiviral medications, that seek to halt further spread of the infection by stopping the cleaving of polyproteins, that are basically the precursors to viral glycoproteins (Xue et al., 2008). This could be the reason behind the potential efficacy of statins as a treatment option for COVID-19. The recent successful attempt at crystallization of the Mpro of SARS-CoV-2 paved the way for a study to investigate the effect of statins on the novel coronavirus. The study measured the affinity of several well-known statins including rosuvastatin, pitavastatin, fluvastatin, and lovastatin, along with a few others, as standard ligands to a certain receptor, that in this case was the SARS-CoV-2 Mpro (Reiner et al., 2020). 
Known previously to have antiviral activity against Ebola virus (Fedson, 2014), statins can bind to the Mpro of SARS-CoV-2 with a higher affinity than that of the protease inhibitors. This is a groundbreaking finding in several contexts, indicating the potential therapeutic value of statins in the treatment of COVID-19, as these cholesterol lowering drugs are possibly capable of stopping the replication of SARS-CoV-2. Arguably, the most optimal results are achieved with pitavastatin, as it binds SARS-CoV-2 Mpro with a binding energy higher than that of the other statins, e.g., rosuvastatin, fluvastatin and lovastatin.

Statins comprise the primary line of treatment for individuals with familial hypercholesterolemia, which is one of several underlying conditions that predispose the patients to acute cardiovascular complications, ultimately putting them at an increased risk of severe COVID-19 (Vuorio, Watts, \& Kovanen, 2020). The anti-inflammatory properties of statins, along with their regulatory effects on the immune system, and potential antiviral activity against SARS-CoV-2, collectively comprise an incontrovertible body of evidence that warrants further clinical research, especially in the case of pitavastatin.

Keywords : statins, Mpro, pitavastatin, SARS-CoV-2, COVID-19

\section{Conflict of Interest Statement}

All authors involved in preparation of this manuscript declare that they have no conflict of interest.

\section{References}

Angus, D. C., \& van der Poll, T. (2013). Severe Sepsis and Septic Shock. New England Journal of Medicine, 369 (9), 840-851. doi:10.1056/NEJMra1208623

Fedson, D. S. (2014). A Practical Treatment for Patients With Ebola Virus Disease. The Journal of Infectious Diseases, 211 (4), 661-662. doi:10.1093/infdis/jiu474

Reiner, Ž., Hatamipour, M., Banach, M., Pirro, M., Al-Rasadi, K., Jamialahmadi, T., . . . Sahebkar, A. (2020). Statins and the COVID-19 main protease: in silico evidence on direct interaction. Archives of Medical Science, 16 (3), 490-496. doi:10.5114/aoms.2020.94655

Sheervalilou, R., Shirvaliloo, M., Dadashzadeh, N., Shirvalilou, S., Shahraki, O., Pilehvar-Soltanahmadi, Y., . . . Nazarlou, Z. (2020). COVID-19 under spotlight: A close look at the origin, transmission, diagnosis, and treatment of the 2019-nCoV disease. Journal of Cellular Physiology, n/a (n/a). doi:10.1002/jcp.29735

Shrivastava-Ranjan, P., Flint, M., Bergeron, É., McElroy, A. K., Chatterjee, P., Albariño, C. G., . . Spiropoulou, C. F. (2018). Statins Suppress Ebola Virus Infectivity by Interfering with Glycoprotein Processing. mBio, 9 (3), e00660-00618. doi:10.1128/mBio.00660-18

Vuorio, A., Watts, G. F., \& Kovanen, P. T. (2020). Familial hypercholesterolaemia and COVID-19: triggering of increased sustained cardiovascular risk. Journal of Internal Medicine, 287 (6), 746-747. doi:10.1111/joim. 13070

Xue, X., Yu, H., Yang, H., Xue, F., Wu, Z., Shen, W., . . Rao, Z. (2008). Structures of Two Coronavirus Main Proteases: Implications for Substrate Binding and Antiviral Drug Design. Journal of Virology, 82 (5), 2515. doi:10.1128/JVI.02114-07 